\title{
Arthroscopic Subacromial Decompression for Small and Medium Size Tears of Rotator Cuff without Tendon Repair
}

\author{
Suhail Karkabi, Nahum Rosenberg \\ Shoulder Service, Rambam-Human Health Campus, Haifa, Israel \\ Email: s karkabi@rambam.health.gov.il
}

Received 20 September 2014; revised 20 October 2014; accepted 17 November 2014

Copyright (c) 2014 by authors and Scientific Research Publishing Inc.

This work is licensed under the Creative Commons Attribution International License (CC BY). http://creativecommons.org/licenses/by/4.0/

(c) (i) Open Access

\section{Abstract}

According to our previous clinical impression, we hypothesized that patients who had symptomatic rotator cuff tendon tear in a diamemter below $3 \mathrm{~cm}$ would benefit from arthroscopic subacromial decompression only, without the need for the repair of the thorn tendon. From 1998 to 2003, 160 patients (168 shoulders) had arthroscopic subacromial decompression for impingement syndrome with a torn rotator cuff without repairing the tear of the cuffs $(120$ males and 40 females). The average patient age was 64 years and the average follow up was 30 months. At follow up, 96 patients $(60 \%)$ had full recovery; 55 patients $(35 \%)$ had residual low grade shoulder pain on effort and 7 patients $(5 \%)$ had poor outcome. In the last group of patients with unsatisfactory outcome, the postoperative Constant score was only 64 points. In $95 \%$ of patients, the average Constant score values raised from average preoperative value of 62 to postoperative average score of 82. According to these results, there is an indication that in patients in the seventh decade of life or older with symptomatic rotator cuff tear, pain relief and good shoulder function can be achieved by more conservative approach of subacromial decompression alone, providing a clear diagnosis that the diameter of the tear in the supraspinatus and infraspinatus tendons is below 3 cm.

\section{Keywords}

Impingement Syndrome, Arthroscopic Subacromial Decompression, Rotator Cuff Tear

\section{Introduction}

In young healthy individuals, the rotator cuff tendons are exposed to high tensile forces without failing or losing

How to cite this paper: Karkabi, S. and Rosenberg, N. (2014) Arthroscopic Subacromial Decompression for Small and Medium Size Tears of Rotator Cuff without Tendon Repair. Open Journal of Clinical Diagnostics, 4, 243-246. 
their structural integrity [1] [2]. With age, these tendons are weakened by degeneration, loose their tensile strength and might be thorn [1] [3]. Additionally, the cuff tendons might degenerate with repetitive mechanical strain [3] [4]. Structural weakness of the rotator cuff tendons is expressed by partial- and full-thickness tears, and when the tear is large enough proximal migration of the humeral head might occur causing a secondary subacromial impingement [5] [6].

Due to increasing use of arthroscopy as the surgical approach in the treatment of shoulder disorders, tears of the rotator cuff tendons and muscles have been thoroughly documented and described. As the consequence, the management of rotator cuff tears has been defined according to tear size, patient age and activity level, and tear etiology [7].

Shoulder subacromial impingement syndrome is one of the most common causes of shoulder pain. The diagnosis of the subacromial impingement is usually clinical and based on the provocative clinical tests [8]-[10], but occasionally might be supported by dynamic imaging, e.g. sonographic examination.

Occasionally, subacromial impingent syndrome coexists with tears in rotator cuff tendons, when one of these two entities is causative of the other. Operative treatment is usually directed for solvation of both problems, i.e. for subacromial decompression followed by rotator cuff repair. But there is challenging evidence that show that this combined approach is not always necessary. Ellman and Kay evaluated the effects of subacromial decompression alone and found a correlation between success of the procedure and size of the rotator cuff tear [11]. In that report, patients with small tears demonstrated $90 \%$ good functional results, while only $50 \%$ of good results were achieved when tears were larger than $2 \mathrm{~cm}$. Similarly, Garts man and Hammermam reported 50\% good results after arthroscopic debridement without repairing the cuff tear [12]. In contradiction, Montgomery et al., who compared the results between arthroscopic decompressions without cuff repair to open repairs of the torn rotator cuff, showed that the patients who were treated with arthroscopic decompression alone had less satisfactory results than those who had additional repair of the cuff [13]. In this report, there was evidence that seventy eight percent of patients after repair of the cuff and subacromial decompression had satisfactory functional outcome in comparison to sixty-one percent of satisfactory outcome in patients treated with arthroscopic subacromialde compression alone. Since our clinical impression was similar to the former cited authors, we hypothesize that patients who have symptomatically moderate in size rotator cuff tendon tear will benefit from arthroscopic subacromial decompression alone, without the need for the repair of the thorn tendon.

\section{Methods}

Between 1998 and 2005, 160 consecutive patients (168 shoulders) underwent arthroscopic subacromial decompression of the shoulders as a treatment for impingement syndrome with small and moderate tears of rotator cuff, up to $3 \mathrm{~cm}$ in diameter [14], in the supraspinatus tendon, without repairing the cuff. The average age of the patients in the study group was 64 years (range 60 to 76 years), 118 men and 40 women.

Rotator cuff tears' diameters were determined arthroscopically.

Fourteen patients with average follow up of 30 months (range 20 to 50 months) were evaluated retrospectively; 158 patients (166 shoulders) were available for a prospective analysis.

The preoperative and postoperative subjective and objective status of the effected shoulders was assessed using the Constant shoulder score [15]. The scale of this score is 0 - 100 points, when 100 points is a normal shoulder in young and fit individual. Additionally, a subjective satisfaction from the procedure outcome was evaluated in post-operative follow up.

Patients with arthroscopic or imaging evidence of intra-articular damage, SLAP or Bankart lesions, labral tears, stiff shoulders and large or massive tears of the cuff were excluded from the study.

\subsection{Surgical Technique}

The arthroscopic subacromial decompression was carried out according to a standard method with the patient in the beach-chair position. In all patients, examination under general or regional anesthesia was performed and compared with the other side. Routine portals were developed and diagnostic arthroscopic examination was performed initially and the glenohumeral joint was inspected for intra-articular pathology. Then the arthroscope was placed into the subacromial space and a complete bursectomy was performed. After complete bursectomy the subacromial space was evaluated, then a subacromial decompression with shavers and bone cutters was performed and the coracoacromial ligament was resected. 


\subsection{Postoperative Management}

The patients were advised to stay in a sling for one week but advised to take their arm out for gentle Codman-Pendulum exercises. After the first week they were instructed to do home exercises and they began formal physical therapy, working with a therapist to regain active and passive range of motion and return back to active function as soon as possible.

The criteria for the cessation of physical therapy were full passive range of motion in all planes. Patients were followed up again until the strength of the affected side was equal to that of the opposite side or, if the non-dominant side was involved, the affected side was within $10 \%$ of the dominant one.

The rehabilitation process was completed during six months post operatively. After the cessation of the rehabilitation treatment the patients were followed on the annual basis.

\section{Results}

All patients in our study had impingement syndrome with small (below $1 \mathrm{~cm}$ in diameter) and moderate $(1-3$ $\mathrm{cm}$ in diameter) tears of supraspinatus, seventy-nine patients with small tears and eighty-nine with moderate tears in supraspinatus tendon.

96 patients (101 shoulders, 62 with small tears and 39 with moderate tears) had excellent outcome, because after the rehabilitation period presented normal function of the effected shoulder and pain-free range of motion.

Fifty-five patients (57 shoulders, 14 with small tears and 43 with moderate tears) had partial functional restraint, i.e. restoring almost normal torque strength and function with mild pain.

In these two groups of patients, the average preoperative Constant score of 62 raised to 82 postoperatively $(\mathrm{p}<0.01)$.

Seven patients (8 shoulders, 3 with small tears and 5 with moderate tears) had poor outcome with persistent pain and limited range of motion. In these patients an unsatisfactory average postoperative Constant score of 64 was evident.

\section{Discussion}

In this report, we describe operative treatment of impingement syndrome in elderly patients with small or moderate tears of supraspinatus by subacromial decompression without repairing the cuff. In this study group, $96 \%$ of the patients had a satisfactory postoperative functional outcome.

Ellman and Kay evaluated the effects of subacromial decompression alone in patients with rotator cuff tears [11] and found a correlation between success of the procedure and size of the tear. Patients with small tears demonstrated $90 \%$ good results, but only $50 \%$ good results were achieved when tears were larger than $2 \mathrm{~cm}$ in diameter. Similarly, Gartsman and Hammerman reported 50\% good results after arthroscopic debridement without repairing the cuff tear in another series of patients [12].

Montgomery et al. compared the results between arthroscopic decompressions without cuff repair to open repairs of the torn rotator cuff [13]. The patients in this study treated with arthroscopic decompression alone had less satisfactory results than those who had repair of the cuff. Seventy-eight percent with repair of the cuff had satisfactory results compared with $61 \%$ of patients treated with arthroscopic decompression without repair.

Our results are similar to the report of the Kempf et al. [16] who analyzed 210 rotator cuff tears treated by arthroscopic acromioplasty alone showing that arthroscopic subacromial decompression without suture of small or moderately sized tears of the cuff in elderly less active lower demand patients over 60 years old with impingement syndrome is an effective method of treatment for their shoulder pain and limitation of function.

The postoperative management of the patients after the acromioplasty alone is less demanding because there is no need to immobilize the shoulder to protect the suture of the repaired cuff. Therefore, this allows almost immediate mobilization without complicity of axillary hygiene. We found this to be of great advantage for the elderly patient.

According to these results, there is an indication that in patients in the seventh decade of life or older with symptomatic rotator cuff tear, pain relief and good shoulder function can be achieved by more conservative approach of subacromial decompression alone, providing there is a clear diagnosis that the diameter of the tear in the supraspinatus and infraspinatus tendons is below $3 \mathrm{~cm}$. This clinical approach might reduce the postoperative burden and facilitate the rehabilitation in patients with described above characteristics. 


\section{References}

[1] Belvins, F.T., Djurasovic, M., Flatow, E.L. and Vogel K.G. (1997) Biology of the Rotator Cuff Tendon. Orthopedic Clinics of North America, 28, 1-16. http://dx.doi.org/10.1016/S0030-5898(05)70260-1

[2] Wilson, C.L. and Duff, G.L. (1943) Pathologic Study of Degeneration and Rupture Supraspinatus Tendon. Archives of Surgery, 3, 121-135. http://dx.doi.org/10.1001/archsurg.1943.01220140003001

[3] Uhthoff, H.K. and Sano, H. (1997) Pathology of Failure of the Rotator Cuff Tendon. Orthopedic Clinics of North America, 28, 31-42. http://dx.doi.org/10.1016/S0030-5898(05)70262-5

[4] Riley, G.P., Harrall, R.L., Constant, C.R., Chard, M.D., Cawston, T.E. and Hazleman, B.L. (1994) Tendon Degeneration and Chronic Shoulder Pain: Changes in the Collagen Composition of the Human Rotator Cuff Tendons in Rotator Cuff Tendonitis. Annals of the Rheumatic Diseases, 53, 359-366. http://dx.doi.org/10.1136/ard.53.6.359

[5] Ogata, S. and Uhthoff, H.K. (1990) Acromial Enthesopathy and Rotator Cuff Tear: A Radiographic and Hystologic Postmortem Investigation of the Coracoacromial Arch. Clinical Orthopaedics and Related Research, 254, 39-48.

[6] Uzaki, J., Fujimoto, S., Nakagawa, Y., Masuhara, K. and Tamai, S. (1988) Tears of the Rotator Cuff of Theshoulder Associated with Pathologic Changes in the Acromion. Journal of Bone and Joint Surgery, American Volume, 70, 12241230.

[7] Lyons, T.R., Savoie F.H. and Field, L.D. (2001) Arthroscopic Repair of Partial-Thickness Tears of the Rotator Cuff. Arthroscopy, 17, 219-223. http://dx.doi.org/10.1053/jars.2001.8017

[8] Neer, C.S. (1983) Impingement Lesions. Clinical Orthopaedics and Related Research, 173, 70-77.

[9] Neer, C.S. (1972)Anterioracromioplasty for the Chronic Impingement Syndrome in the Shoulder: A Preliminary Report. Journal of Bone and Joint Surgery, American Volume, 54, 41-50.

[10] Hawkins, R.J. and Abrams, J.S. (1992) Impingement Syndrome in the Absence of Rotator Cuff Tear. Orthopedic Clinics of North America, 18, 373-382.

[11] Ellman, H., Kay, S.P. and Wirth, M. (1993) Arthroscopic Treatment of Full Thickness Rotator Cuff Tears: 2 to 7 Years Follow-Up Study. Arthroscopy, 9, 195-200. http://dx.doi.org/10.1016/S0749-8063(05)80374-3

[12] Gartsman, G.M. and Hammerman, S.M. (1997) Full-Thickness Tears: Arthroscopic Repair. Orthopedic Clinics of North America, 28, 83-98. http://dx.doi.org/10.1016/S0030-5898(05)70267-4

[13] Montgomery, T.J., Yerger, B. and Savoie, F.H. (1994) Management of Rotator Cuff Tears: A Comparison of Arthroscopic Debridement and Surgical Repair. Journal of Shoulder and Elbow Surgery, 3, 70-78. http://dx.doi.org/10.1016/S1058-2746(09)80113-4

[14] Liu, S.H. (1994) Arthroscopically Assisted Rotator Cuff Repair. The Journal of Bone \& Joint Surgery (British Volume), 76, 592-595.

[15] Constant, C.R. and Murly, A. (1987) A Clinical Method of Functional Assessment of the Shoulder. Clinical Orthopaedics and Related Research, 21, 160-164.

[16] Kempf, J.F., Geyze, P., Bonnomet, F., Walch, G., Mole, D., Frank, A., Beaufils, P., Levigne, C., Rio, B. and Jaffe A. (1999) A Multicenter Study of 210 Rotator Cuff Tears Treated by Arthroscopic Acromioplasty. Arthroscopy, 15, 56-66. http://dx.doi.org/10.1053/ar.1999.v15.015005 
Scientific Research Publishing (SCIRP) is one of the largest Open Access journal publishers. It is currently publishing more than 200 open access, online, peer-reviewed journals covering a wide range of academic disciplines. SCIRP serves the worldwide academic communities and contributes to the progress and application of science with its publication.

Other selected journals from SCIRP are listed as below. Submit your manuscript to us via either submit@scirp.org or Online Submission Portal.
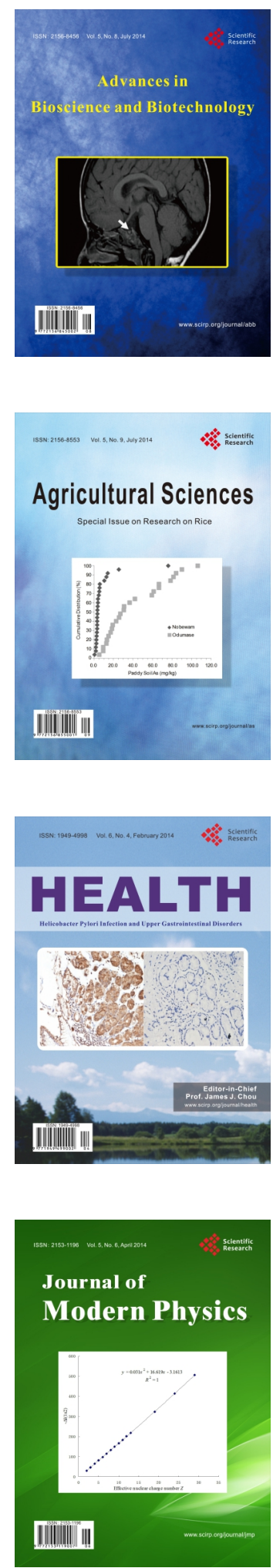
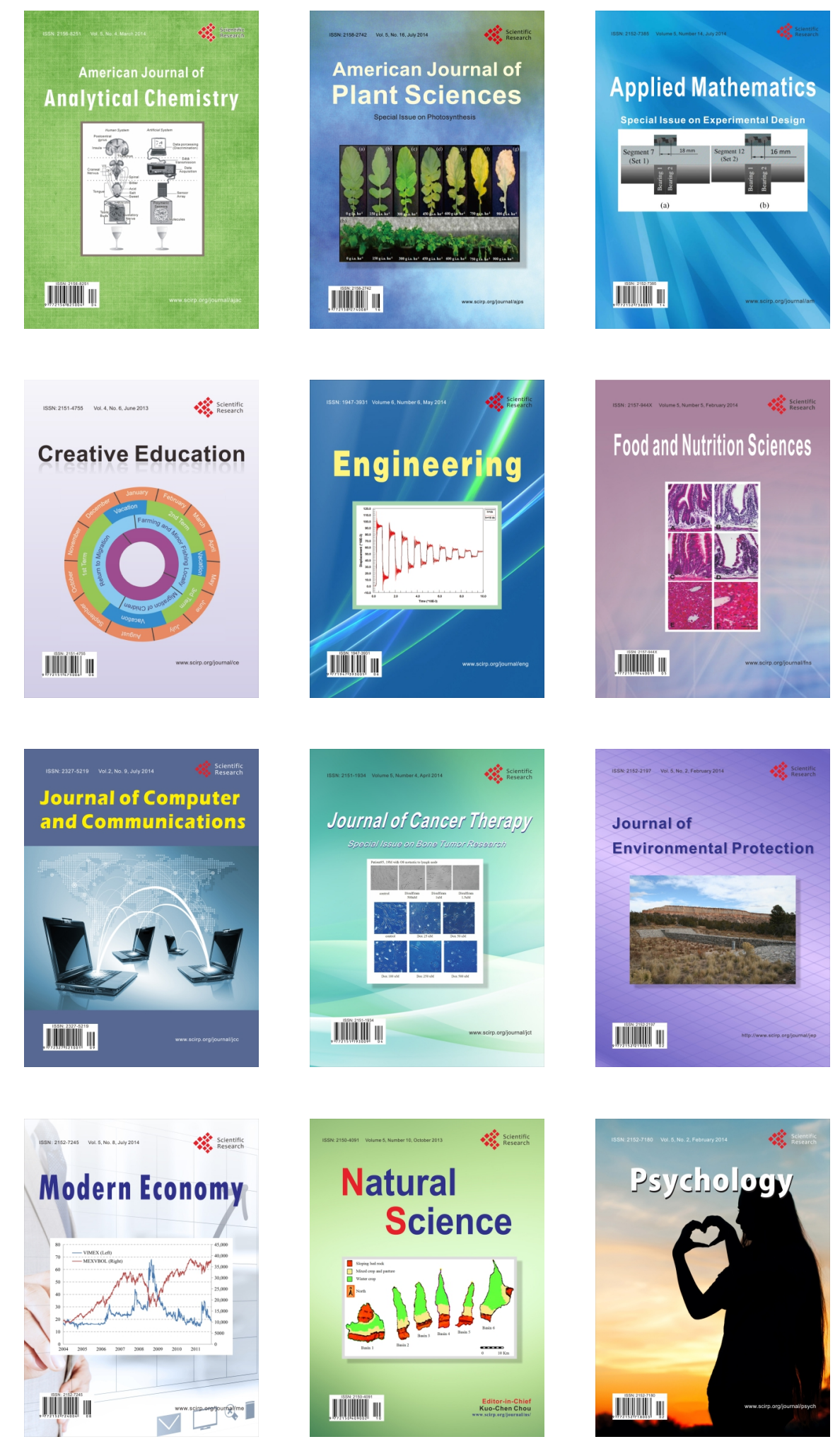